\title{
A co-simulation method for system-level simulation of fluid- structure couplings in hydraulic percussion units
}

\author{
Håkan Andersson ${ }^{1,2}$ (D) $\cdot$ Peter Nordin $^{3} \cdot$ Thomas Borrvall $^{4} \cdot$ Kjell Simonsson $^{2}$ • \\ Daniel Hilding ${ }^{4} \cdot$ Mikael Schill $^{4} \cdot$ Petter Krus $^{3} \cdot$ Daniel Leidermark $^{2}$
}

Received: 3 December 2015 / Accepted: 8 August 2016 / Published online: 29 August 2016

(C) The Author(s) 2016. This article is published with open access at Springerlink.com

\begin{abstract}
This paper addresses a co-simulation method for fluid power driven machinery equipment, i.e. oil hydraulic machinery. In these types of machinery, the fluid-structure interaction affects the end-product performance to a large extent, hence an efficient co-simulation method is of high importance. The proposed method is based on a 1D system model representing the fluid components of the hydraulic machinery, within which structural 3D Finite Element (FE) models can be incorporated for detailed simulation of specific sub-models or complete structural assemblies. This means that the fluid system simulation will get a more accurate structural response, and that the structural simulation will get more correct fluid loads at every time step, compared to decoupled analysis. Global system parameters such as fluid flow, performance and efficiency can be evaluated from the 1D system model simulation results. From the 3D FE-models, it is possible to evaluate displacements, stresses and strains to be used in stress analysis, fatigue evaluation, acoustic analysis, etc. The method has been implemented using two well-known simulation tools for fluid power system simulations and FE-simulations, respectively, where the interface between the tools is realised by
\end{abstract}

Håkan Andersson

hakan.andersson@se.atlascopco.com

1 Construction Tools PC AB, Dragonvägen 2, 39127 Kalmar, Sweden

2 Division of Solid Mechanics, Linköping University, 581 83 Linköping, Sweden

3 Division of Fluid and Mechatronic Systems, Linköping University, 58183 Linköping, Sweden

4 DYNAmore Nordic AB, Brigadgatan 5, 58758 Linköping, Sweden use of the Functional Mock-up Interface standard. A simple but relevant model is used to validate the method.

Keywords Co-simulation · Fluid-structure coupling · System simulation · Functional mock-up interface $\cdot$ Fluid power machinery $\cdot$ Transmission line modelling

\section{Introduction}

During the last years, the development of commercial software suits for simulating Fluid-Structure Interaction (FSI) problems has been substantial, and today FSI-functionality exists in many simulation environments, e.g. LS-DYNA [1] and ANSYS [2]. These FSI-methods are based on cosimulation of Finite Element Method (FEM) and Computational Fluid Dynamics (CFD) models. The utilisation of FSI-methods can today be found in advanced industrial fields such as aerospace, nuclear, defense and medicine, see e.g. [3, 4]. Examples from the machine building industry are unusual, but an exception is found in [5], where an FSI analysis of the valve system in a reciprocating air compressor is performed. The 3D-models (fluid or structural) in the existing applications are relatively small and often represent only one part of the global system model to keep the computational requirements acceptable with respect to available resources. Global 3D FSI-simulations on system level have not been found in the literature and, if any, are likely rare. This can be explained by the vast computational effort that is needed not only for solving the problems, but also for the post-processing of long simulated time periods. One FSI-application for an impeller pump can, however, be found in [6] where a strong coupling between a highly flexible impeller and the fluid flow was prevailing, and a method for quasi-steady simulations was developed. Within 
the general machine building industry, time and computational resources required for large-scale FSI-analysis are generally not available and other FSI-methods, that are less computationally demanding, are needed. It has been found that system simulation models, that are described by Ordinary Differential Equations (ODE), or 1D Partial Differential Equations (PDE), are often used to simulate the functional behaviour of a product, i.e. to study the interaction between different components and subsystems, while high fidelity methods such as CFD or FEM are used to study phenomena at a much more detailed level. However, by combining them the system simulation models can be used to provide the proper boundary conditions at a low computational cost.

There are applications where a 1D system model is cosimulated with a 3D model, structural or fluid, to incorporate global system behaviour into the latter model. Such applications are often single domain simulations, i.e. fluid domain or structural domain, and consist of two simulation models that communicate through a co-simulation interface; e.g. a 1D system model of ODE and a CFD-model [7] or a Multi Body Simulation (MBS) model and a 3D Finite Element (FE) model [8].

In this paper, a different approach, a multi-domain method, that incorporates the fluid-structure coupling into the simulation model for fluid power systems is presented. This method is based on FEM for the structural part of the model and 1D system simulation for the fluid part. The communication between the simulation models is based on the Functional Mock-up Interface (FMI) standard [9]. One big advantage here is that the fluid simulation is computationally very inexpensive, but still capable of representing the desired dynamic behaviour. This is particularly important for simulations over long time periods. Since the models are coupled, it is possible to evaluate the response from all fluid- and structural loads.

The current methodology that is used during the product development phase for hydraulic percussion units is based on decoupled analyses of the fluid and the structural systems. The fluid loads are simulated in a 1D system simulation model, extracted as quasi-static maximum data and then transferred as a load case matrix to a number of static 3D FE-analyses. Compared to the current methodology, the proposed method has several advantages, where one of the most important would be the dynamic coupling between the fluid and the structure. One challenge has been to define and implement such a co-simulation interface that meets the requirements on accuracy and stability for coupled analyses where wave transmission is crucial. Wave propagation and transmission in both fluid and structure are of great importance for hydraulic percussion units and a co-simulation interface must be able to handle this phenomenon. Other challenges were to design the interface so that it is flexible and easy to use by engineers, and to allow co-simulation across a computer network since computationally demanding simulations often run on dedicated computers.

A simple, but relevant, example of a hydraulic percussion unit is modelled and simulated to show the advantages and potential of the proposed method. Such a unit, that can be found in power tools, rock drills, hydraulic hammers and similar equipment, consists of a number of complex mechanisms whose behaviour is hard to capture in an uncoupled analysis due to the strong interaction of the fluid and mechanical parts. The example, described in more detail in Sect. 4, focuses on the pressurisation of fluid cavities as a means to control the percussive motion of a piston. The fluid pressure will act on the piston surfaces and thereby result in a force that affects the motion.

The outline of the paper is as follows. The proposed simulation method is presented and the implemented cosimulation interface is described in detail. The application example and three different co-simulation cases are then explained and the accuracy of the method is evaluated and compared to an equivalent system-level simulation model. Finally, the results are discussed.

\section{Proposed simulation method}

The need for an alternative simulation method during the product development phase of fluid power machinery becomes evident when, e.g. it is necessary to increase the simulation time to reach structural steady-state conditions when evaluating fatigue, and/or for acoustical analysis of reciprocating hydraulic machinery. The problems arise when the hydraulic percussion unit is to be analysed for several working strokes and the fluid-structure coupling directly affects the timing of the motion in the unit.

The proposed method, illustrated in Fig. 1, consists of a 1D fluid system model, a structural 3D FE-model and a co-simulation interface. The method uses the system model as the simulation master, while the FE-model is used as a detailed sub-model, which is represented by a Functional Mock-up Unit (FMU).

FMI is a standardised interface for facilitating intercommunication between different simulation tools. The standard supports both model-exchange and co-simulation interoperability. In the model-exchange mode, a simulation model is exported in a standardised format so that other simulation tools can execute it directly using their own solvers. In this work, different solution techniques are required and the co-simulation mode must be used instead. In this mode, each simulation tool is active during the simulation in solving its own part. An FMU is a module that wraps a model with an interface based on the FMI standard, 
Fig. 1 Overall simulation method sequence

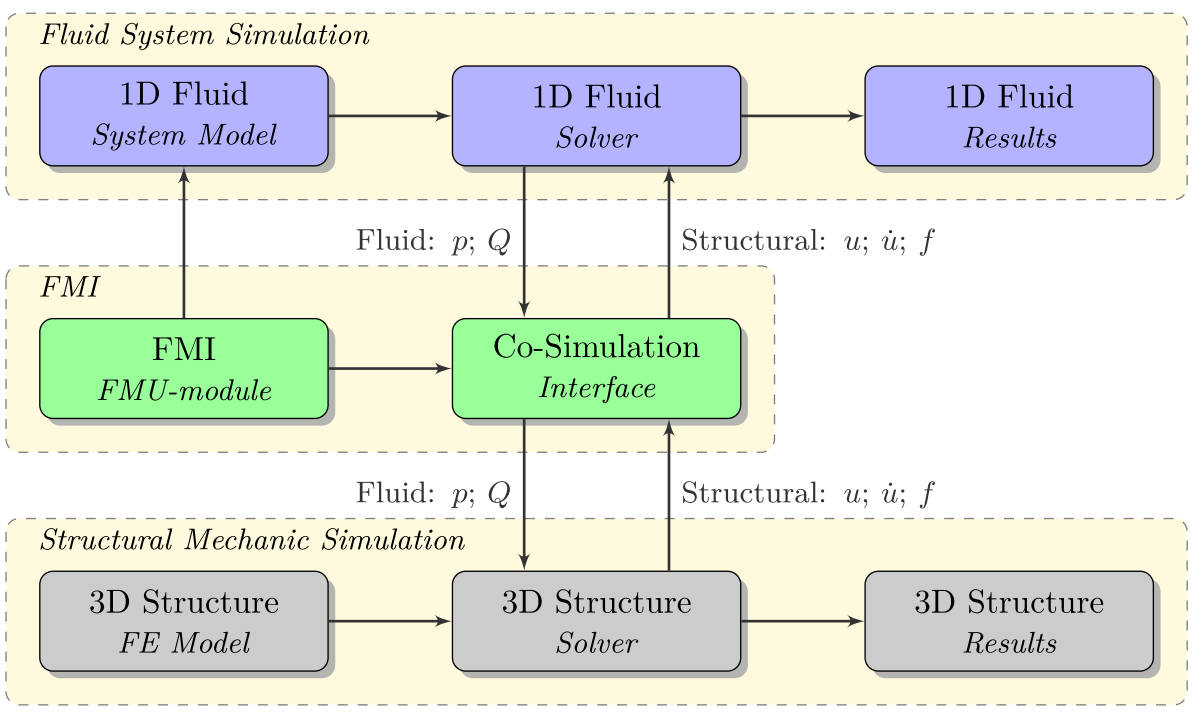

Fig. 2 Example of a multidomain 1D system model. The translational movement of a hydraulically powered piston is converted into a rotation. The measured angle of the end shaft is feedback into a control system for a valve that directs the flow into the piston

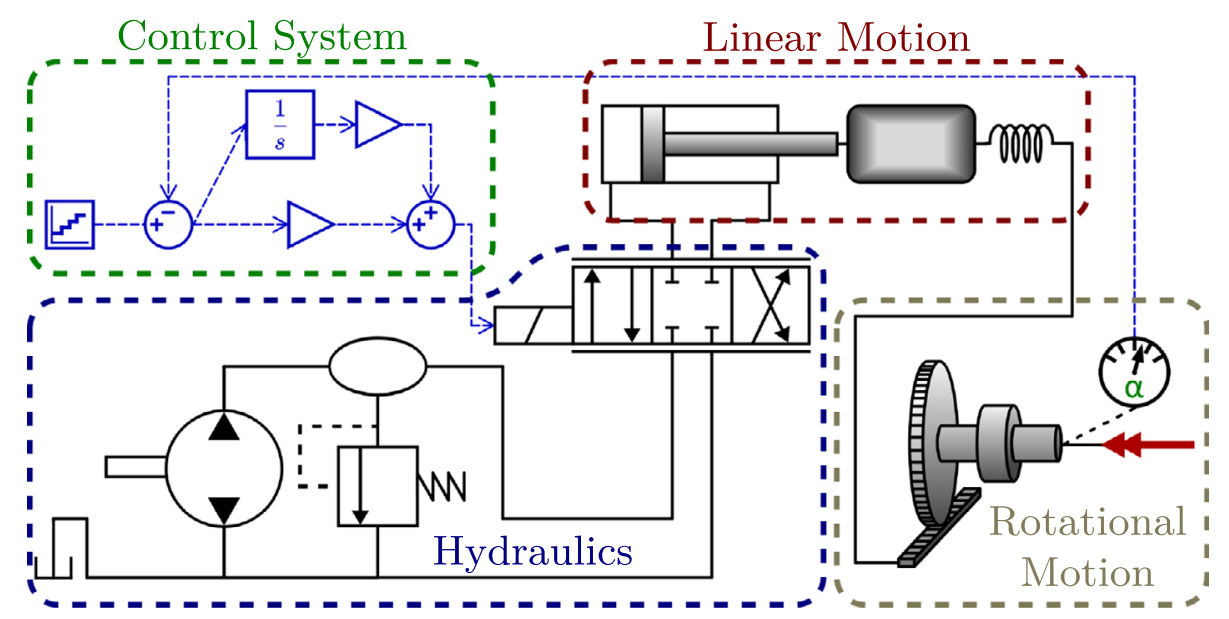

thereby making it possible to execute it in different FMI supporting simulation tools. Typically, an FMU is exported from one tool and is then imported into the other. A co-simulation FMU is a compressed archive that essentially consists of a pre-compiled dynamically linkable library with a C-code interface and an Extensible Markup Language (XML) file containing the FMI description. The description specifies among other things the input and output variables and the parameters that are available. The use of a C-code interface and plain-text XML description files makes the FMU portable to almost any platform and tool.The 1D system model consists of a number of sub-models describing different components in the hydraulic fluid system, e.g. valves, cavities, orifices, etc. Furthermore, 1D sub-models from other domains, such as 1D mechanical mass-springdamper components, can also be included. A multi-domain system model example is shown in Fig. 2.

In the FE-model, the fluid system is represented as actions on pressurised surfaces. From the fluid system model, the pressure $p$ is delivered to the FE-model, and vice versa the displacement $u$, velocity $\dot{u}$ and mechanical force $f$ are received, see Fig. 1. Since these values must be scalars in the 1D system, the average displacements and velocities over the pressurised surfaces are used.

At the end of the simulation, the fluid domain variables are available and system properties such as performance and efficiency can be evaluated. From the FE-results, it is possible to evaluate displacements and velocities of the $3 \mathrm{D}$ geometry. These can be used for instance as input data for acoustic analyses. The stresses and strains are also available, e.g. for stress analysis and structural fatigue evaluation.

\section{Implementation}

The proposed method has been implemented using the open-source 1D system-simulation program Hopsan [10, 11], for simulation of the hydraulic fluid components and 
the commercial FE-software LS-DYNA [1], for the structural part. Hopsan uses the Transmission Line Modelling (TLM) method for modelling of physical components, which has been shown to be very suitable for simulation of hydro-mechanical systems [12]. These tools were chosen because each of them has several advantages when simulating hydraulic percussion units. The TLM method used in Hopsan captures the pressure waves generated in the fluid system during operation in a realistic way and it is also in this case very fast compared to iterative solver techniques. The explicit solver in LS-DYNA works well when analysing stress waves in solid structures or when simulating contacts between structural parts, which are important mechanisms in a hydraulic percussion unit.

The Hopsan simulation tool can import FMUs, but LS-DYNA currently has no FMI export capability. For this reason, an FMU that communicates with LS-DYNA through the LS-DYNA user-defined function (UDF) code has been developed. The UDF for applying custom external loads is used as the entry point into the FE-software and it is accessed through a TCP/IP socket communications library. This library is used on both the FMU and UDF sides, and it was implemented using the $\mathrm{C}$ programming language since $\mathrm{C}$-functions can be called directly from the Fortran language code used in LS-DYNA. This means that no new interface technology needs to be implemented on the LS-DYNA side. On the system model side, the FMU is imported and the use of TCP/IP makes it possible to run the system model and FE-simulation on different computers. This means that dedicated simulation computers or compute clusters can be used to increase the simulation performance.

From a users perspective, it would be cumbersome to manually synchronise the development of both system model, FE-model, UDF routine and FMI interface description. To hide the low-level workings of Hopsan, LS-DYNA UDF and FMI from the user, a custom-made configuration file based on the native Keyword format in LS-DYNA and an automatic FMU generator was developed. The user only needs to specify the interface points on the model level: essentially, which ports in the system model that should represent which nodes or segments in the FE-model, and what unit conversions that are necessary. The co-simulation connectivity and work flow are illustrated in Fig. 3.

Connecting 1D and 3D simulation models has also been done using other tools. In [13], a method for incorporating PDE models into OpenModelica [14] is presented. The example considered regards control of the temperature in a copper block, where the controller, modelled using the Modelica language, is simulated in OpenModelica and the heat equation is solved using the open-source simulation tool HiFlow3 [15] with an FE-approach. The FMI standard is used as the interface between the two programs, an FMU

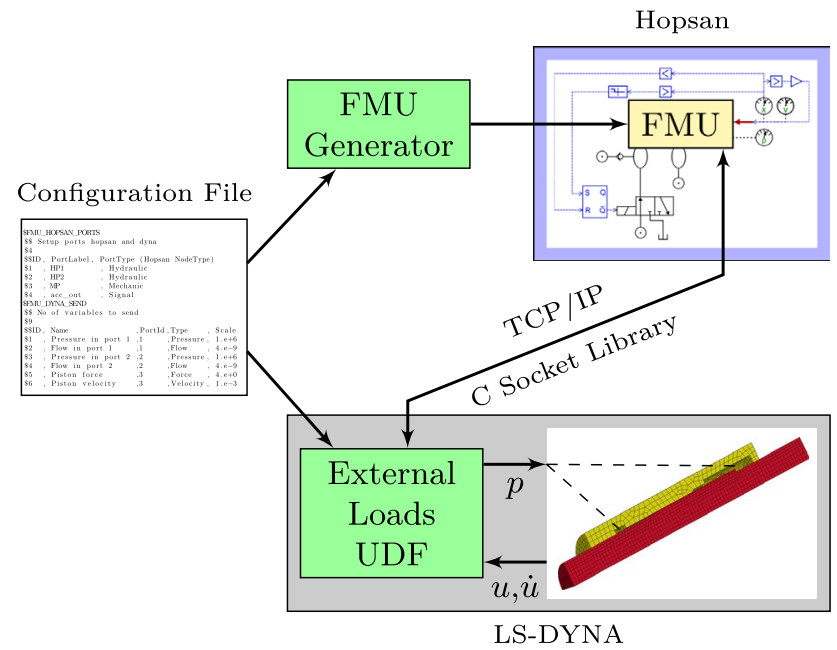

Fig. 3 Overview of the co-simulation work flow

is exported from HiFlow 3 and imported directly into the OpenModelica model.

Another example can be found in [16], where a fluid domain co-simulation of a building energy supply system is investigated. In this case, the Dymola simulation tool was used to simulate a solar thermal collector and storage. The majority of the system, including solar collector, pipes, heat exchanger and controller were modelled and simulated as a differential algebraic equation system but the thermal storage, a hot water accumulator, was simulated in greater detail using CFD analysis in ANSYS. The models were adapted so that the same physical part was simulated in both programs at the interface points. Essentially, parts of the pipes connected to the accumulator were simulated in CFD so that the interface points represented 1D to 3D pipe simulation. The TLK inter software connector (TISC) [17] was used as the interface framework. TISC uses TCP/ IP sockets in a client and server framework to interconnect different simulation programs. The proposed method in this paper is also based on socket communication but since only two programs are connected, peer-to-peer direct communication is sufficient.

In [18], a 1D pipe and valve system and a detailed 3D CFD pump model are coupled. In this case, the fluiddomain simulation tools are driven by external code and data are exchanged using text files. At each iteration, the $1 \mathrm{D}$ system is solved at steady state and the $3 \mathrm{D}$ model is simulated until convergence within the iteration step is reached. This differs from the proposed method in this paper, which relies on explicit solvers in both programs, i.e. each program takes one step at a time.

A crash analysis comparison, in the structural domain, between a full FE-model and an FE-model in which one part had been replaced by a 1D co-simulated model was 
Fig. 4 A TLM element, illustrated by a pipe, introduces a time delay $(T)$ of the effort $(p)$ and flow $(Q)$ variables entering on either side. Equations 1, 2 and 3 give the TLM boundary equations

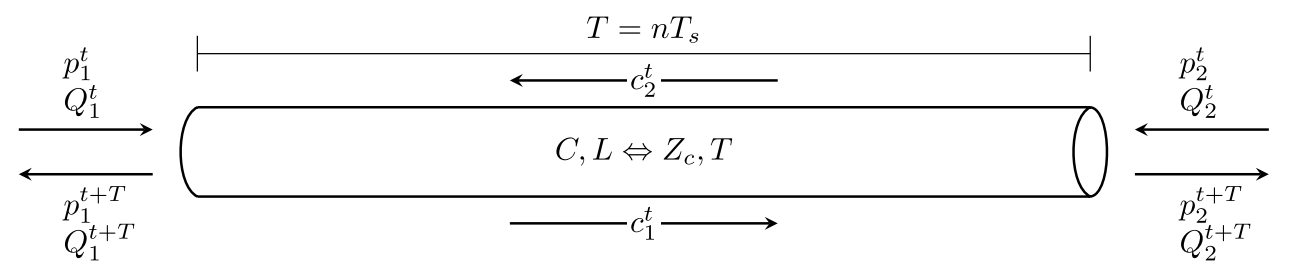

made in [19]. It was possible to greatly shorten the simulation time in this way, while keeping the deviation from the more detailed complete FE-simulation low. Identifying which parts of a model that are suitable for simplified 1D or detailed 3D modelling is an important step when using this type of co-simulation methodology.

Co-simulation between LS-DYNA and either Microsoft Office Excel [20] or Matlab/Simulink [21] was shown in [22]. Two example applications were considered: control of an inverted pendulum and control of an automatic seat belt safety system during a frontal crash of a car. To define the communication points, an external list was used to specify the type of variable (position, translational/rotational velocity or acceleration) and what nodes in the FE-model they would represent. Similarly to the approach proposed in this study, this list was then used as a configuration file by the communications module connecting the two programs.

\subsection{The TLM method}

The TLM method was applied to modelling and simulation of distributed physical systems in [23], where it was referred to as bi-lateral delay line modelling. In short, the method is based on the one-dimensional wave equation and it introduces time-discrete model elements, TLM elements, that effectively decouple different parts of the model. This allows each separated part to be simulated individually, because they only depend on delayed values from the other side of the elements. The time delays ensure that the interface points will be weakly coupled and the method, therefore, makes it possible to solve systems using explicit methods rather than needing implicit solvers for large equation systems. In a simulation, the shortest possible delay is the same as the simulation time step $T_{s}$. Longer delays can be realised by delaying values multiple time steps. The number of steps to use depends on the physical properties of the object being modelled, primarily its length and wave propagation speed.

The TLM method has also been used in [24] where an efficient meta-model-based co-simulation framework for mechanical systems is presented. The focus of the framework is on co-simulation of axis and ball-bearing joint applications, where both translational and rotational mechanic TLM couplings are defined. Their idea is to simulate each part of a system model in the tool that is the most suitable, but to use TLM elements at the interface points to decouple the different environments. Care is taken to insert the elements where natural physical time delays exist between the parts. Another example is [25] where the method was used in co-simulation of a complete wheelloader. Matlab/Simulink was used to model the dieselengine and transmission, Adams MBS for the tires, frame and mechanical load and Hopsan, for the hydraulic control system. A similar work connecting Hopsan to Adams for simulation of a forester machine is found in [26]. Here, the co-simulation interface is also realised by the FMI interface, and Adams is administering the simulation process. The hydraulic system is modelled in Hopsan and exported as an FMU that is imported directly into Adams. Further, a comparison between different co-simulation integration methods was made in [27]. The conclusion was that stability could be improved if the simulation models to be coupled were divided into two groups, simulated after one another. The TLM method was one of the methods compared, and TLM elements made up one of these groups. This method proved to have superior stability properties, but a model approximation, a so-called parasitic inductance, that depends on the length of the simulation time step was introduced. When choosing the simulation time step, it is important to be able to quantify this approximation. An example of how to calculate the relevant parasitic inductance for the TLM elements used is covered later in this section.

For the co-simulation example in this paper, loss-less, single-time-step TLM elements are used. It is assumed that the components only undergo small deformation and that linear-elastic material properties can be used. Internal friction is also neglected in the elements, but it is possible to lump frictions contributions to the boundaries [28] if they have a meaningful effect. The loss-less bi-lateral delay element is illustrated in Fig. 4. The equations describing it, derived for the time-domain [29], are given in Eqs. 1, 2 and 3 where $p_{1}$ and $p_{2}$ are the "effort" variables and $Q_{1}$ and $Q_{2}$ are the "flow" variables at the respective boundary. During the delay duration, compression (fluid) or strain/stress (solid) waves represented by $c_{1}$ and $c_{2}$ travel in the respective directions through the element. The loss-less elements characteristic impedance $Z_{\mathrm{c}}$ is a scalar property that relates the physical properties: capacitance $C$ and inductance $L$, to the introduced time delay $T$. 


$$
p_{2}^{t+T}=\overbrace{p_{1}^{t}+Z_{c} Q_{1}^{t}}^{c_{1}^{t}}+Z_{c} Q_{2}^{t+T}
$$

$p_{1}^{t+T}=\underbrace{p_{2}^{t}+Z_{c} Q_{2}^{t}}_{c_{2}^{t}}+Z_{c} Q_{1}^{t+T}$

$Z_{c}=\sqrt{\frac{L}{C}}$

$T=\sqrt{L C}$

As is shown by the last term in Eq. 1, the characteristic impedance acts as a damping together with the boundary velocities. This fact can be used in co-simulation if the simulation method on the receiving end supports damping control. A typical example is in MBS where spring-massdamper types of sub-models are common, see e.g. [26]. Directly affecting the damping was, however, not possible in the LS-DYNA implementation presented here, and an approximation had to be made. To calculate the pressure acting on the piston, prior to the FE-simulation step, the damping term was based on the previous flow value according to Eq. 4. For short time steps, the difference should not be that large, but this is nevertheless a possible source of error that has not been investigated further in this paper.

$p_{2, \text { approx }}^{t+T}=c_{1}^{t}+Z_{c} Q_{2}^{t}$

Material or fluid properties determine $C$ and $L$, while $T$ equals the simulation time step for a single-step delay element. The fact that $Z_{c}, T, L$ and $C$ all depend on each other means that a choice must be made whether to model a TLM element so that the physical inductance, capacitance or characteristic impedance is preserved. In the hydraulic cavities, there is a small amount of fluid that does not move much; the dynamics is mainly affected by the fluid elasticity. The large inductive contribution comes from moving the relatively heavy piston. In that case, modelling the correct inductance (mass) would be important to preserve the dynamic response. For stationary parts, the capacitance (stiffness) is of more interest. The cavities are, therefore, modelled as purely capacitive, elastic fluid springs.

In a hydraulic percussion unit, the typical material properties of interest are those of the fluid and solid elements (piston). For the hydraulic TLM element, used as the co-simulation interface on the fluid side in this work, the Purely Capacitive model $Z_{c}^{\mathrm{PC}}$ is given by Eq. 5 , where $K$ is the bulk modulus and $V$ the volume of the fluid cavity. To cancel out the inductance, Eqs. 2 and 3 are combined. The resulting model will then preserve the desired capacitance (stiffness) regardless of the time delay $T$. For comparison, the characteristic impedance for a capacitive element representing the piston is given in Eq. 6. In this model, linear elasticity and a 1D simplification (no lateral expansion) is assumed. Material and component properties of interest are: initial cross-sectional area $A_{0}$, initial length $X_{0}$ and Young's modulus $E$. In the mechanical case, capacitance is the same as $1 / k$, where $k$ is equal to stiffness, and the inductance is the same as the mass.

$Z_{c, \text { fluid }}^{\mathrm{PC}}=\frac{T}{C_{\text {fluid }}}=\frac{K}{V} T \quad\left[\frac{\mathrm{Pa} \cdot \mathrm{s}}{\mathrm{m}^{3}}\right]$

$Z_{c, \text { piston }}^{\mathrm{PC}}=\frac{T}{C_{\text {piston }}}=T k=T \frac{E A_{0}}{X_{0}} \quad\left[\frac{\mathrm{N} \cdot \mathrm{s}}{\mathrm{m}}\right]$

It is rarely possible to select $T$ and set $Z_{c}$ so that $L$ and $C$ perfectly coincide with real physical values. The delay $T$ in each TLM element will cause an individual modelling error called parasitic inductance or parasitic capacitance. The ideal situation in TLM simulation is when $T$ corresponds to $T_{\text {physical }}$, the real physical wave propagation timedelay of the modelled component. As a comparison, using the values from Table 2 with steel for the piston and oil for the fluid, the wave speed is $\sim 5172$ and $\sim 1341 \mathrm{~m} / \mathrm{s}$, respectively. For a single-step line, one meter long, the optimal simulation time step would be $T_{\text {opt,piston }} \approx 1.9 \times 10^{-4} \mathrm{~s}$ and $T_{\text {opt,fluid }} \approx 7.5 \times 10^{-4} \mathrm{~s}$. As the computer simulation is discrete by nature, the modelled delay $T$ depends on the chosen simulation time step $T_{s}$, and the number of delay steps in the line, $T=n T_{s}$. The wave speed through the line (the speed of sound), together with the time step used, results in a perceived length of the line model. When this length is too long, then additional parasitic inductance (extra mass) will be added. At the same time, parasitic capacitance will cause the modelled stiffness to decrease. For the purely capacitive model of the hydraulic cavities, all model approximation will show up as a parasitic inductance according to Eq. 7. To represent the hydraulic inductance as its mechanical counterpart mass with unit $\mathrm{kg}$, it is multiplied with the affected area in square, according to Eq. 8.

$L_{\text {fluid,percived }}=T Z_{c}^{\mathrm{PC}}=\frac{T^{2}}{C}=\frac{T^{2} K}{V} \quad\left[\frac{\mathrm{Pa} \cdot \mathrm{s}^{2}}{\mathrm{~m}^{3}}\right]$

$L_{\text {mechanic }}=A_{\text {affected }}^{2} \cdot L_{\text {fluid }} \quad[\mathrm{kg}]$

A comparison of the model approximation (parasitic mass) for different time steps for a fluid cavity with volume $2 \times 10^{-4} \mathrm{~m}^{3}$, affecting an area $8 \times 10^{-4} \mathrm{~m}^{2}$ and a piston with diameter $0.07 \mathrm{~m}$ and length $0.4 \mathrm{~m}$, is given in Table 1 . The size parameters are relevant to the application example and the material properties are listed in Table 2. Both the fluid cavity and the piston are modelled as purely capacitive, and thus any time delay larger then zero will give 
Table 1 A comparison of the parasitic inductance, in unit kg, Eqs. 7 and 8 , for the purely capacitive fluid cavity model in Eq. 5 and the piston model in Eq. 6, for different simulation time steps

\begin{tabular}{llllll}
\hline Time delay $(T)$ & \multicolumn{3}{l}{ Fluid cavity } & & \multicolumn{2}{l}{ Piston } \\
\cline { 2 - 3 } \cline { 5 - 6 } \cline { 5 - 6 } & Inductance & $Z_{\mathrm{c}}$ & & Inductance & $Z_{\mathrm{c}}$ \\
\hline $1.0 \mathrm{E}-07$ & $5.12 \mathrm{E}-08^{\mathrm{a}}$ & $8.0 \mathrm{E}+05$ & & $2.020 \mathrm{E}-05$ & 202.0 \\
$5.0 \mathrm{E}-07$ & $1.28 \mathrm{E}-06$ & $4.0 \mathrm{E}+06$ & & 0.001 & 1010.2 \\
$1.0 \mathrm{E}-06$ & $5.12 \mathrm{E}-06$ & $8.0 \mathrm{E}+06$ & & 0.002 & 2020.4 \\
$5.0 \mathrm{E}-06$ & $1.28 \mathrm{E}-04$ & $4.0 \mathrm{E}+07$ & & 0.051 & 10102.2 \\
$1.0 \mathrm{E}-05$ & $5.12 \mathrm{E}-04$ & $8.0 \mathrm{E}+07$ & & 0.202 & 20204.4 \\
$5.0 \mathrm{E}-05$ & 0.0128 & $4.0 \mathrm{E}+08$ & 5.051 & 101021.8 \\
$7.733661734 \mathrm{E}-05$ & 0.0306 & $6.2 \mathrm{E}+08$ & & $12.084^{\mathrm{a}}$ & 156253.8 \\
$1.0 \mathrm{E}-04$ & 0.0512 & $8.0 \mathrm{E}+08$ & & 20.204 & 202043.7 \\
$5.0 \mathrm{E}-04$ & 1.2800 & $4.0 \mathrm{E}+09$ & 505.109 & 1010218.4 \\
$1.0 \mathrm{E}-03$ & 5.1200 & $8.0 \mathrm{E}+09$ & 2020.437 & 2020436.8 \\
$5.0 \mathrm{E}-03$ & 128.0000 & $4.0 \mathrm{E}+10$ & 50510.919 & 10102183.9 \\
\hline
\end{tabular}

${ }^{a}$ Marks the best time step choice for the two models for the listed time delays

Table 2 The fluid and solid material properties used in the simulations

\begin{tabular}{lllll}
\hline Quantity & Material & Symbol & Unit & Value \\
\hline Bulk modulus & Oil & $K$ & $\mathrm{GPa}$ & 1.6 \\
Density & Oil & $\rho_{\text {oil }}$ & $\mathrm{kg} / \mathrm{m}^{3}$ & 890 \\
Elastic stiffness & Steel & $E$ & $\mathrm{GPa}$ & 210 \\
Poissons ratio & Steel & $v$ & & 0.3 \\
Density & Steel & $\rho_{\text {steel }}$ & $\mathrm{kg} / \mathrm{m}^{3}$ & 7850 \\
\hline
\end{tabular}

parasitic inductance. Due to the small volume of the cavity, the time step should be kept below $10^{-4} \mathrm{~s}$ to avoid adding noticeable parasitic mass in relation to the piston weight. The piston has a mass in reality, and there is a specific simulation time step when the parasitic mass coincides with the actual mass $(12.08 \mathrm{~kg})$. It is clear that different TLM element models have different optimal simulation time steps. In general, the user must choose the lowest required time step that give acceptable approximations and then use multi-step delay models for the longer components. The co-simulation interface will not handle this automatically.
Another source of approximation is that the cavity volumes have been modelled as fixed. In reality, the volumes will change when the piston moves. Equation 7 shows that the smaller the volume, the larger the parasitic inductance will become. In this case, the approximate volume of the cavities lies in the range $\left[1.3 \times 10^{-4}, 2.5 \times 10^{-4}\right] \mathrm{m}^{3}$ which at a time step $10^{-4} \mathrm{~s}$ would represent a parasitic mass range of $[0.04,0.08] \mathrm{kg}$. At this and shorter time steps, the difference is negligible.

Note that the piston model in Eq. 6 is only used for the parasitic mass comparison here. The purpose of the presented method is to allow higher fidelity simulation of the solid materials, and it would not make sense to use a simplified TLM model in this case. This simplified model should not be confused with the co-simulated FE piston model analysed later in this paper.

\subsection{The Hopsan simulation tool}

The Hopsan simulation tool is a dedicated 1D TLM simulation software used primarily for simulation of fluid power and mechatronic systems. Physical systems are modelled using the TLM method and power ports are used as the interface between sub-models. A power port defines the variables that are necessary to describe power transfer in a specific domain. In Hopsan, they also contain other variables particular to that domain and the TLM method. Examples of available power ports for 1D power transfer are: hydraulic, translational mechanic, rotational mechanic, electric and pneumatic types. The use of power ports makes it possible to define physically motivated connection points similar to the ones that exist in the real world. For example, a hydraulic cylinder has hydraulic connections for the fluid pressure supply and a mechanical attachment on the piston that transfers the force to some load as shown in Fig. 5. Aside from power-port modelling, the program also supports scalar signal flow for arbitrary mathematics. Port types are then of input or output type. For the co-simulation method described, only the hydraulic and translational mechanic port types are considered.

In Hopsan, the TLM elements are referred to as C-type sub-models, since they calculate the characteristic waves. The boundary values of the element are solved by the
Fig. 5 In Hopsan, the physical sub-models of C- or Q-type are connected pairwise to solve Eq. 9. The power ports contain the TLM and domain-specific variables

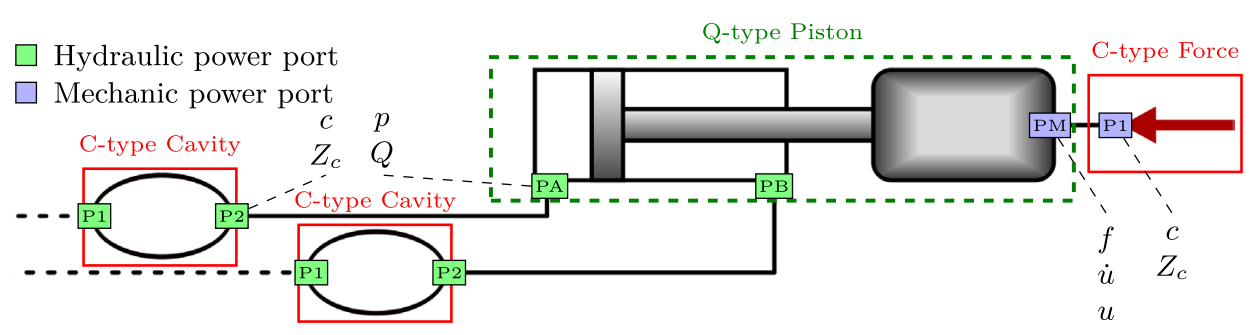


so-called Q-type sub-models that calculate the flow variable $Q$. These two types are responsible for solving one part each of the TLM equation as illustrated by Eq. 9, and must therefore be connected pairwise as Fig. 5 shows. This grouping approach is the same as was recommended in [27]. The C-type models will introduce the approximations discussed previously, and must be implemented so that they calculate the characteristic waves. The Q-type models can be more freely implemented, and these usually contain their own internal integrators. For instance, the piston in Fig. 5 is essentially a mass with two surfaces subjected to fluid pressure given by $c$ and $Z_{c}$. It internally integrates the resulting accelerations into velocity (the flow variable $Q$ ) and position. Essentially, this is the same thing that happens in LS-DYNA, where the FE-model of the piston is simulated, and it is therefore suitable to let the FMU cosimulation module be of Q-type.

$$
\underbrace{p_{2}^{t+T}}_{\text {Q-type }}=\underbrace{c_{1}^{t}+Z_{c}}_{\text {C-type }} \underbrace{Q_{2}^{t+T}}_{\text {Q-type }}
$$

\subsection{LS-DYNA user-defined function}

The general non-linear ODE, i.e. the equation of motion, that is solved by LS-DYNA is given by Eq. 10 .

$\mathbf{M u ̈}+\mathbf{C u}+\mathbf{f}_{\text {Int }}=\mathbf{f}_{\text {Ext }}$

where $\mathbf{M}$ is the mass matrix, $\mathbf{C}$ is the damping matrix and $\mathbf{f}_{\text {Int }}$ is the internal force vector. The external force vector, $\mathbf{f}_{\mathrm{Ext}}$, represents all external loads acting on the FE-model. The displacement $\mathbf{u}$ is solved explicitly by the central difference time integration scheme described by Eqs. 11 and 12. For further description regarding the explicit integration scheme, see e.g. [30].

$\dot{\mathbf{u}}^{n+\frac{1}{2}}=\dot{\mathbf{u}}^{n-\frac{1}{2}}+\ddot{\mathbf{u}}^{n} \Delta t^{n}$

$\mathbf{u}^{n+1}=\mathbf{u}^{n}+\dot{\mathbf{u}}^{n+\frac{1}{2}} \Delta t^{n+\frac{1}{2}}$

The UDF functionality for adding external loads $\mathbf{f}_{\text {Ext }}$, is used as the interface on the LS-DYNA side. The input variables ( $c$ and $Z_{c}$ ), representing the pressure, from the system model are used to calculate these forces and the user-defined configuration file specifies on which locations they are to be applied. The resulting displacements and velocities are sent back from the FE-model to the system model. This procedure is repeated each time step until the analysis is completed.

\subsection{Co-simulation interface}

To avoid needless complication of the FE-model, it is represented by a sub-model of Q-type in Hopsan, essentially as a replacement to the piston model in Fig. 5. This leaves the TLM specific modelling of the characteristic impedance and wave variables in Hopsan. The Q-type FE-model will determine the flow variables, in this case the velocity and the position (by integration), based on the input from the neighbouring C-type components in the system model. This results in a natural interface point between the models. In the application example, the hydraulic cylinder presented in Sect. 4, the hydraulic oil cavities (fluid-domain) or mechanic external forces are implemented as TLM elements in Hopsan. The fluid pressure or the external forces are then applied to the structure-domain piston surface segments and contribute to the external forces vector $\mathbf{f}_{\text {Ext }}$ in Eq. 10.

Since Hopsan is administering the simulation, it will trigger each simulation step in LS-DYNA. Hopsan uses fixed time step simulation and the FE-simulation is set to use the same step size. Technically, it would be possible to sub step the simulation in LS-DYNA, but that is deemed to be of little value as the time spent on the 1D system simulation is negligible compared to the FE-simulation time. The co-simulation sequence and synchronisation are shown in Fig. 6, where the UDF module in LS-DYNA is called at the beginning of each FE time step to set the external forces. The two programs exchange variables at this point. The LS-DYNA simulation loop always waits for input at the beginning of each step. The socket receive calls are blocking (with a failure timeout) and this effectively synchronises the simulation steps between the programs. Aside from communicating the simulation variables, control flags are also transmitted. This allows one program to notify the other when the simulation has been finished or otherwise terminated.

\section{Simulations and results}

A simple fluid power model of a hydraulic percussion unit was setup to validate the proposed co-simulation method. This model, at different levels, reflects the main features of such a unit and especially the short duration dynamics in the fluid and the structural systems. A reference model was setup in Hopsan, where the fluid system consists of a hydraulic cylinder, two fluid volumes, a valve and pressure sources, see Fig. 7a. The valve controls the oil flow, while Cavity A and B represent the fluid properties of the oil volumes inside the cylinder, also shown in Fig. 8. The fluid properties used for the hydraulic oil can be found in Table 2. A point mass, representing a rigid body, is connected to the piston part of the hydraulic cylinder. The valve is controlled by a network of logical components, the piston control circuit, in Fig. 7a. Signals are generated at pre-determined piston positions to achieve the piston 
Fig. 6 The co-simulation sequence and simulation step synchronisation between Hopsan and LS-DYNA. The receive points block execution until data have been received

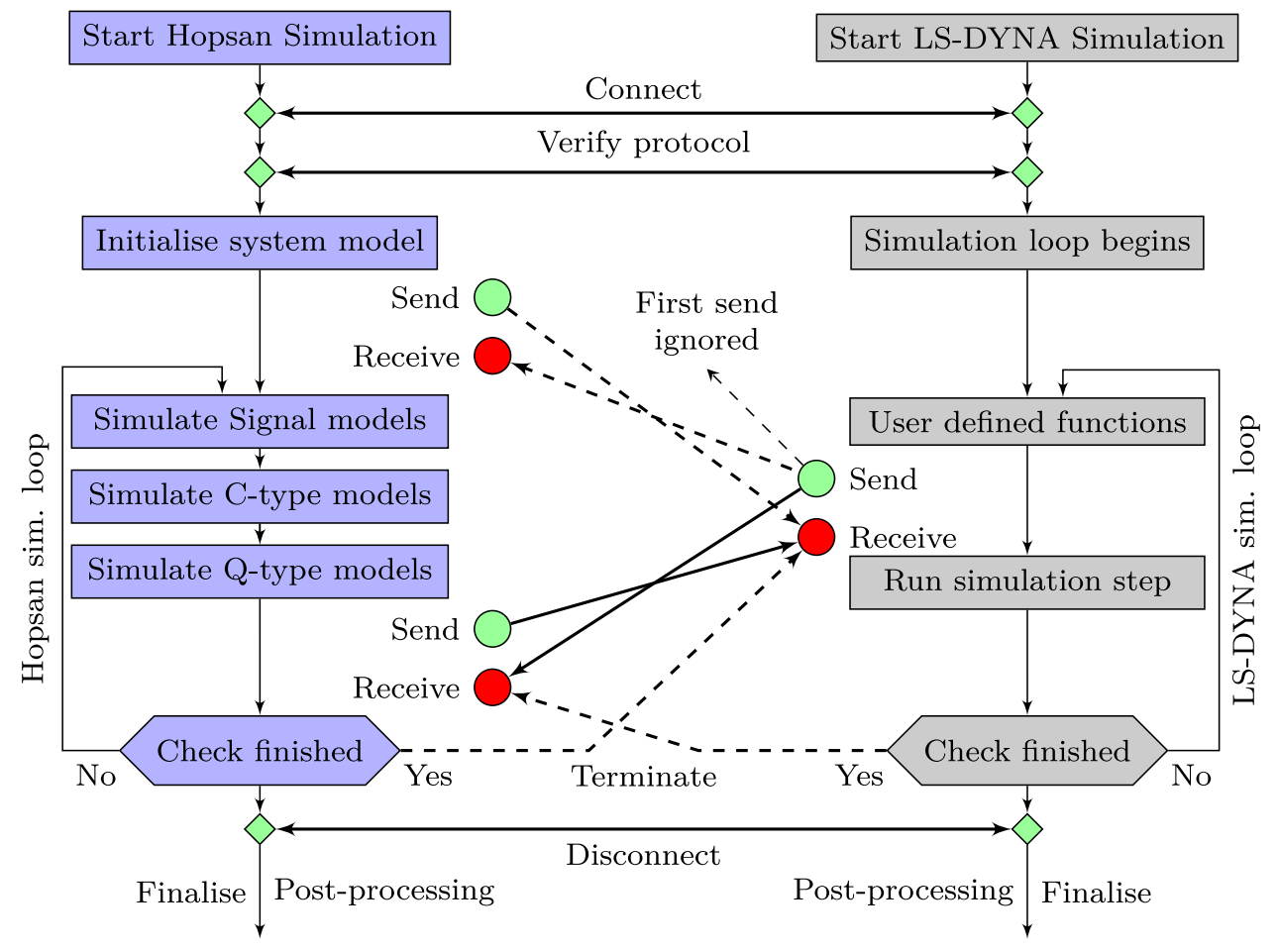

reciprocal movement. The force control circuit activates and deactivates the mechanical force when the piston reaches a certain position. Three sensors were used to collect data during the simulation. The displacement and the velocity sensors are connected to the piston and the pressure sensor is connected to Cavity A. The reference system model is completely simulated in Hopsan and its results are used as a reference during the evaluation of the co-simulation results. A second system model was defined for the co-simulation approach, see Fig. $7 \mathrm{~b}$. This is identical to the reference model, except for the hydraulic cylinder and the force control circuit. The cylinder has been replaced by the imported FMU and the force control circuit is removed since the force is applied inside LS-DYNA. This system model uses the FE-model shown in Fig. 8 during co-simulation with LS-DYNA.

The FE-model of the hydraulic cylinder assembly consists of the piston and the cylinder housing. The mesh consists of 7112 8-node hexahedral solid elements, 5144 for the piston and 1968 for the cylinder housing. Full integration scheme was used for all solid elements. Typical elastic material properties for steel were used in the FE-model, see Table 2. Due to symmetry, a quarter model is used, and the associated boundary conditions are applied to the nodes on the symmetry plane. The piston is free to move in the axial direction but, due to symmetry, constrained in all the other directions. The axial movement of the cylinder housing is constrained due to the fixed boundary condition on the end surface and the other directions are constrained due to symmetry. The total piston mass is defined by the FE-mesh and the material density. Since a quarter FE-model is used a scaling routine implemented in the co-simulation interface was utilised to get the equivalent full model response on the Hopsan side. The piston has two hydraulic control surfaces, $\mathrm{A}$ and $\mathrm{B}$, that are associated with its respective cavities. The pressure, based on $c$ and $Z_{c}$, from the Hopsan simulation is applied to all element segments that belong to each cavity. A control routine keeps track of when the piston element segment is inside or outside the cavity and removes the pressure if the segment is outside.

The start values for the simulation, at $t=0 \mathrm{~s}$, were as follows: piston position $u=0.03 \mathrm{~m}$, piston velocity and acceleration, $\dot{u}=\ddot{u}=0$, pressure in Cavity $\mathrm{A}$ and $\mathrm{B}$, $p=10^{5} \mathrm{~Pa}$.

To evaluate the proposed method, with the co-simulation interface, three different simulation cases with increasing complexity have been investigated. These are:

Case 1 Hydraulically controlled piston with rigid piston and cylinder housing.

Case 2 Case 1 extended with a mechanical force for evaluating the feedback from the structural simulation.

Case 3 Hydraulically controlled piston with linear elastic material properties for piston and cylinder housing materials. Repeated impacts against an elastic cylinder are analysed. 


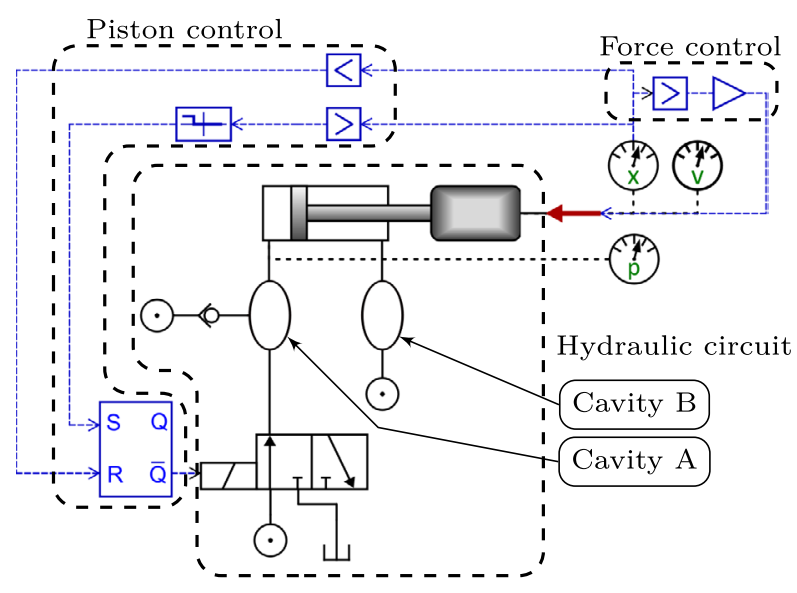

(a) Hopsan reference sytem model

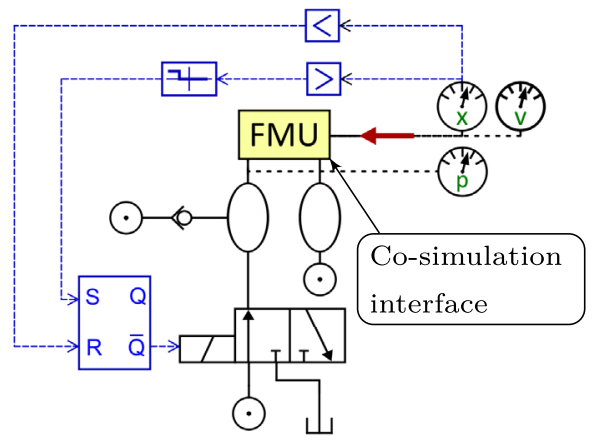

(b) Hopsan system model for co-simulation

Fig. 7 System models for Hopsan simulations. The hydraulic circuit, the piston control and the control of the mechanical force are circled by dashed lines. The meters are sensors for different quantities: $X$ displacement $u, V$ velocity $\dot{u}$ and $p$ pressure. $X$ and $V$ are Hopsan standard nomenclature, but the latter ones will be used throughout this work. The displacement and the velocity sensors are connected to the piston and the pressure sensor is connected to Cavity A in the hydraulic cylinder

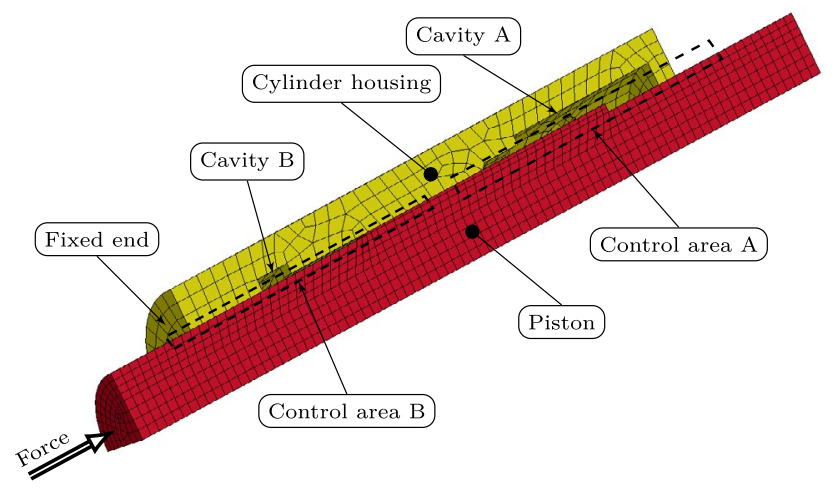

Fig. 8 The quarter FE-model of the hydraulic cylinder assembly that is used in Case 1 and 2. The mechanical force is applied perpendicular to the left end surface of the piston
Case 1 represents a moving mass that is driven by hydraulic pressure and no external force is active. In Case 2, a transient mechanical force is introduced on the structural side to evaluate the feedback from the FE-simulation. This functionality emulates that the piston hits an elastic material at a certain position. The mechanical force is realised through the SENSOR keywords, (*SENSOR_), in LS-DYNA. Using this functionality, a position sensor for the piston was defined. When the piston reaches a pre-set position, the pre-defined mechanical force is activated that reverses the motion of the piston. The force is deactivated when the piston again reaches the pre-set position. Case 3 gives a demonstration of the method when somewhat more realistic conditions, such as elastic materials and real contact definitions, are used. This makes it possible to simulate deformations, stresses and strains. For Cases 1 and 2, the co-simulation results are investigated and compared with the results from the reference model in Hopsan.

The simulations were run on an Intel Xeon E5-1603 (2.8 $\mathrm{GHz}, 4$ core) workstation with 32 GB RAM under Windows 7 (64-bit). Four processor cores with shared memory were used for the LS-DYNA analyses and one core for the Hopsan analysis. Both Hopsan and LS-DYNA were run on the same computer.

\subsection{Case 1}

This case essentially integrates the effect of the hydraulic forces acting on the piston mass, i.e. the mechanical force is set to zero. The time step $T=10^{-6} \mathrm{~s}$ was used here based on a convergence study of the Hopsan reference model. In this study, $T$ was decreased until the amplitude error $\epsilon$ was within acceptable levels. $\epsilon$ was defined according to Eq. 13, where $u_{T_{1}}$ is the piston displacement from the simulation at time step $T_{1}$. RMS represents the Root-MeanSquare value. Piston displacements were extracted from simulations made with two different time step values, e.g. $T_{1}=10^{-4} \mathrm{~s}$ and $T_{2}=10^{-5} \mathrm{~s}$. The time step was decreased one decade at a time and an acceptable level was reached for $T_{1}=10^{-6} \mathrm{~s}$ where $\epsilon<0.02 \%$.

$\epsilon=\frac{\operatorname{RMS}\left(u_{T_{1}}\right)}{\operatorname{RMS}\left(u_{T_{2}}\right)}$

The volume was $8.63 \times 10^{-6} \mathrm{~m}^{3}$ and $2.05 \times 10^{-6} \mathrm{~m}^{3}$, for Cavity $\mathrm{A}$ and $\mathrm{B}$, respectively. The characteristic impedances $Z_{c \text {,fluid }}$ were retrieved for each cavity from Hopsan; $3.09 \times 10^{8} \mathrm{~Pa} \mathrm{~s} / \mathrm{m}^{3}$ and $8.68 \times 10^{8} \mathrm{~Pa} \mathrm{~s} / \mathrm{m}^{3}$. The parasitic masses added due to parasitic inductance were estimated to be $1.4 \times 10^{-4} \mathrm{~kg}$ and $3.3 \times 10^{-5} \mathrm{~kg}$. This amount of parasitic mass can be neglected compared to the actual piston mass in the model $(11.2 \mathrm{~kg})$. The computational time for the co-simulation was approximately $8 \mathrm{~min}$ and for the 


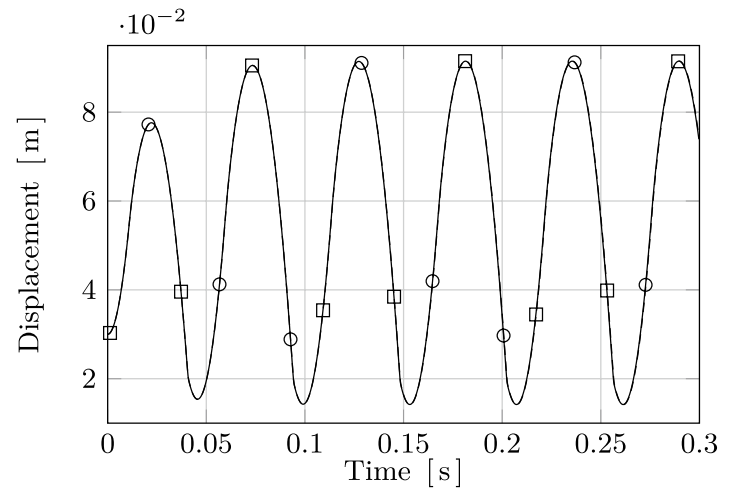

(a) Piston displacement

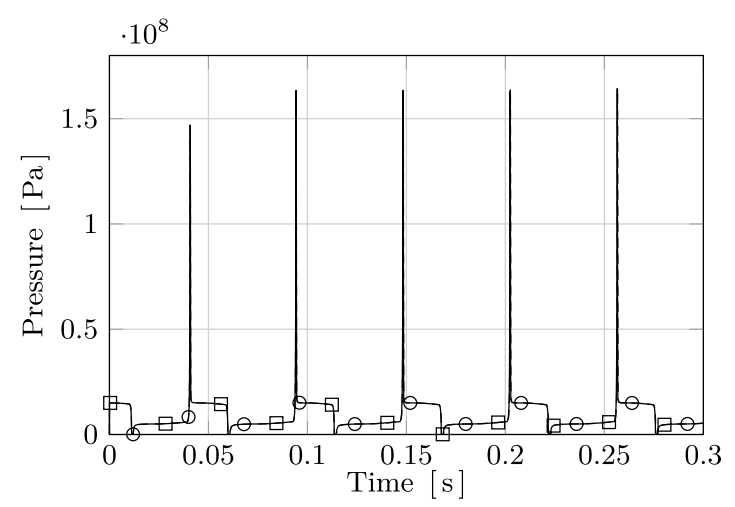

(c) Pressure in Cavity A

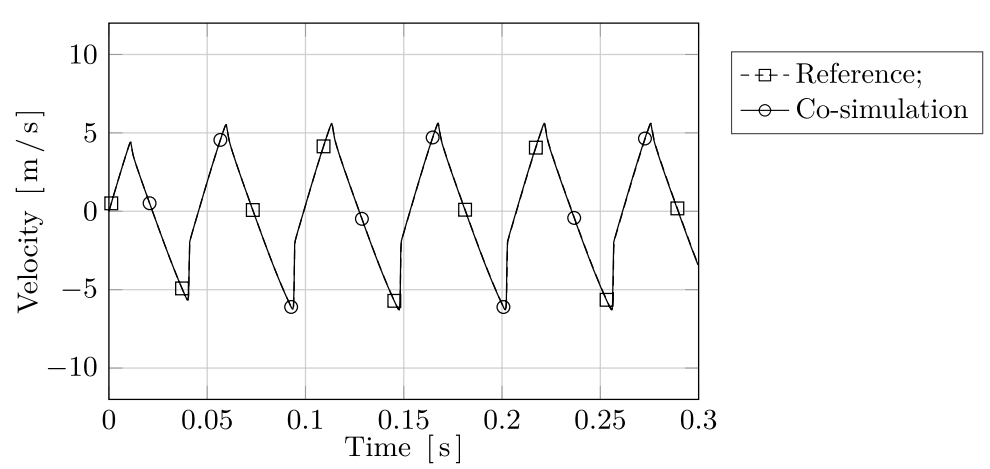

(b) Piston velocity

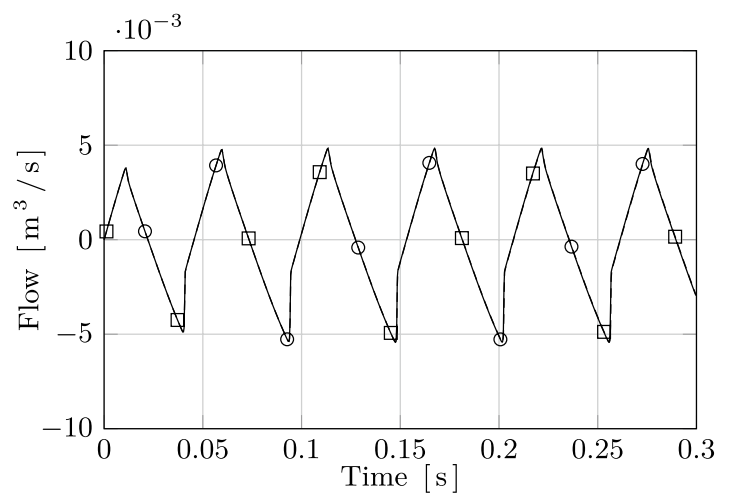

(d) Flow into Cavity A

Fig. 9 Simulation results from Case 1, showing the difference between the reference and the co-simulation models

pure Hopsan reference model $0.3 \mathrm{~s}$. Simulation results are shown in Fig. 9.

Figure $9 \mathrm{a}, \mathrm{b}$ shows that the piston moves in and out of the cylinder housing at a maximum speed of $\sim 5 \mathrm{~m} / \mathrm{s}$. The results shows an identical behaviour for the reference and the co-simulation model. This was to be expected since the two-system models are the same except for the piston movement simulation. In Fig. 9c, it can be seen that the curves are right on top of each other, but when the curves are examined at a greater magnification, a maximum time shift of $\sim 0.13 \mathrm{~ms}$ can be noticed at the last pressure peak, see Fig. 10. It was also found that the magnitude of the time shift is increasing throughout the simulation.

The pressure curve, Fig. 9c, shows that the simulation method is able to handle short duration dynamics in the fluid system, causing pressure peaks of high amplitude and a duration of approximately $0.5 \mathrm{~ms}$. The results also show that the piston reaches a steady-state behaviour after two working cycles, which implies that this method is stable for this simulation model. The FE-results in this case consist of the piston movement and the forces that are acting on the piston.

\subsection{Case 2}

The second case is used to evaluate the simulation method when there is a transient mechanical force acting on the FE-model. The force is here used to simulate a simplified elastic impact, and will affect the pressure and flow in the hydraulic circuit. The magnitude of the force was chosen arbitrarily to $10^{7} \mathrm{~N}$ to give a reasonable behaviour of the system, and its value thus has no direct physical basis. The time step used was $10^{-7} \mathrm{~s}$ resulting in an estimated amplitude error of $<7.5 \%$ based on a convergence study in the same way as in Case 1. A significant part of this error can be related to the modelling of the mechanical force, where the time step has a large influence on the duration of the force.

The volume of the fluid cavities has here been changed to achieve a more realistic behaviour of the hydraulic percussion unit. The volumes that were used for Cavity A and B were: $8.63 \times 10^{-4} \mathrm{~m}^{3}$ and $2.05 \times 10^{-4} \mathrm{~m}^{3}$, respectively. As in Case 1, the characteristic impedances were retrieved from Hopsan; $3.09 \times 10^{5} \mathrm{~Pa} \times \mathrm{s} / \mathrm{m}^{3}$ and $8.68 \times 10^{5} \mathrm{~Pa} \mathrm{~s} / \mathrm{m}^{3}$. The parasitic masses were estimated to be $1.4 \times 10^{-8} \mathrm{~kg}$ 


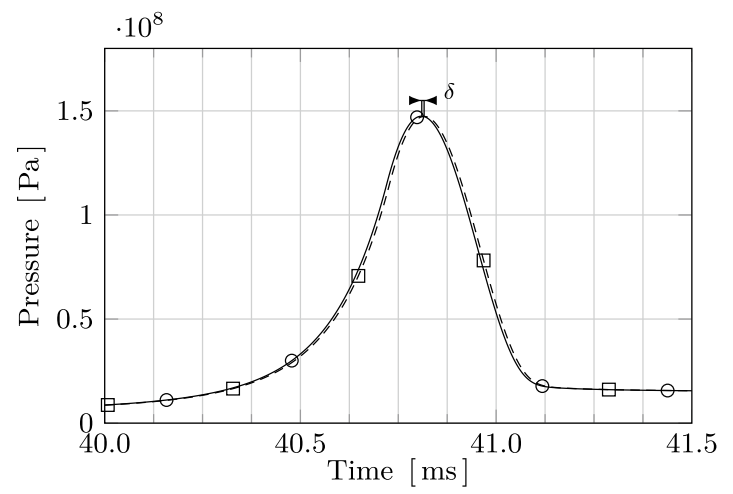

(a) First pressure peak

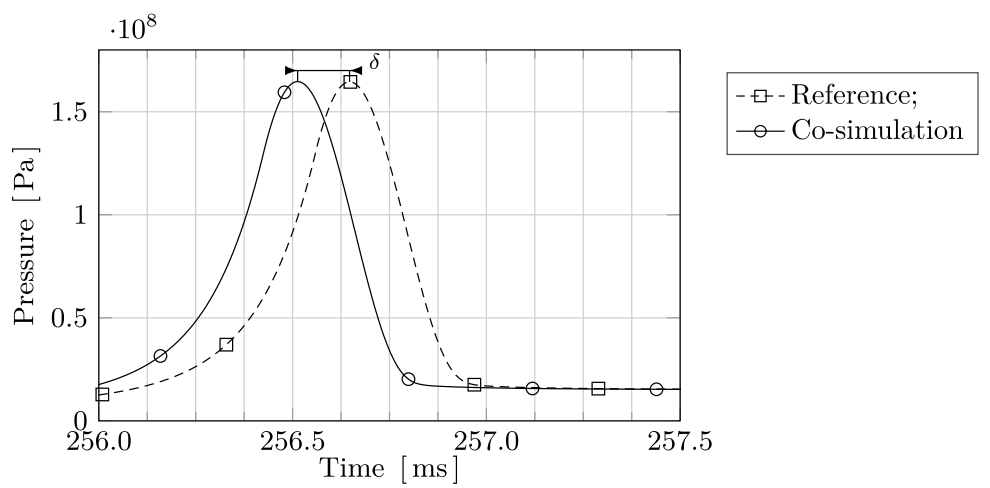

(b) Last pressure peak

Fig. 10 These curves are the same as in Fig. 9c but shown at a much smaller time scale. A time period of $1.5 \mathrm{~ms}$ is displayed around a the first and $\mathbf{b}$ the last pressure peak. The parameter $\delta$ denotes the time shift between the two simulation systems

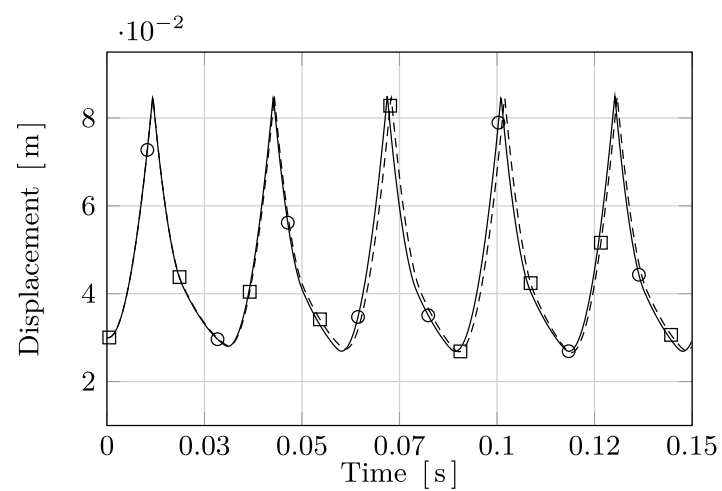

(a) Piston displacement

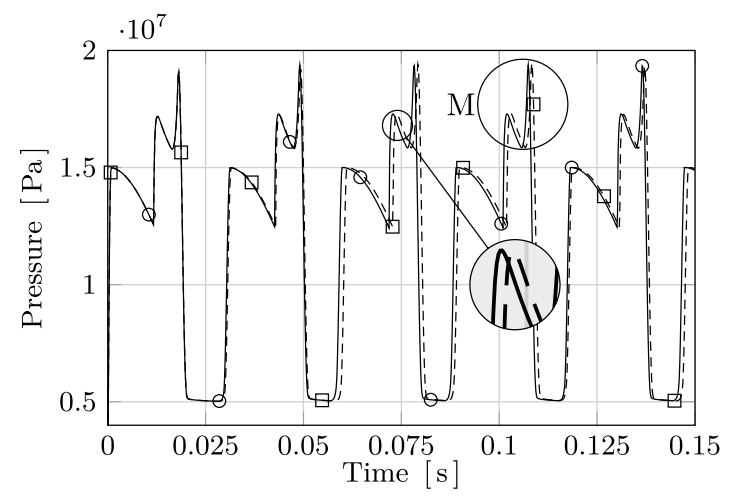

(c) Pressure in Cavity A

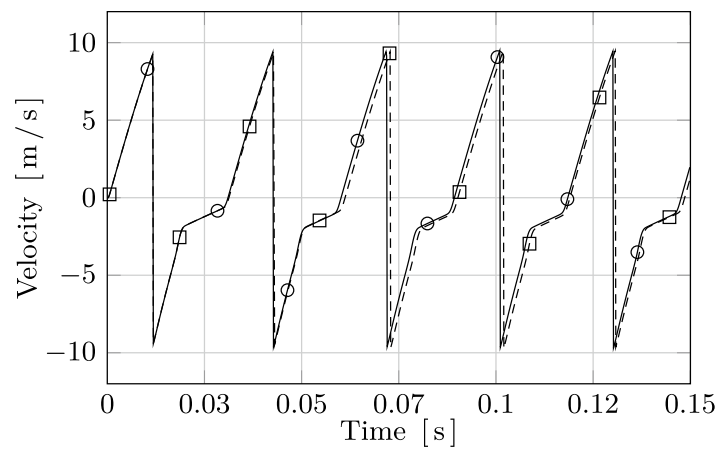

(b) Piston velocity

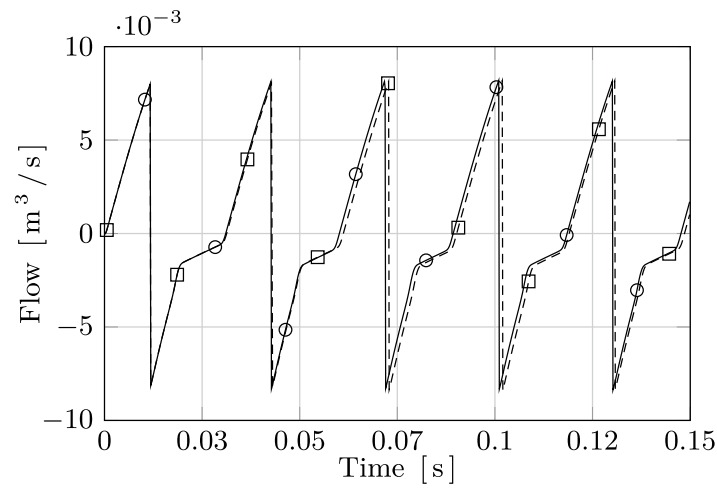

(d) Flow in Cavity A

Fig. 11 Simulation results from Case 2, showing the difference between the reference and the co-simulation models. Region M indicates the pressure behaviour at the time of impact

and $3.3 \times 10^{-9} \mathrm{~kg}$, which are even lower than in Case 1 and can be considered negligible. The computational time for the co-simulation was approximately $29 \mathrm{~min}$ and for the pure Hopsan reference model $0.8 \mathrm{~s}$; the simulation results can be seen in Fig. 11.
The piston displacement curve, Fig. 11a, shows the piston working sequence. At a position of $0.03 \mathrm{~m}$, the piston reaches the inner turning point in the cylinder housing and then it starts to accelerate outwards in the cylinder due to the pressure in Cavity A. When the piston reaches 
Fig. 12 The FE-model used in Case 3. The hydraulic cylinder is the same as in Case 1 and 2, except for the elastic material properties. The left cylindrical part represents the tool. The left end of the tool is rigidly fixed

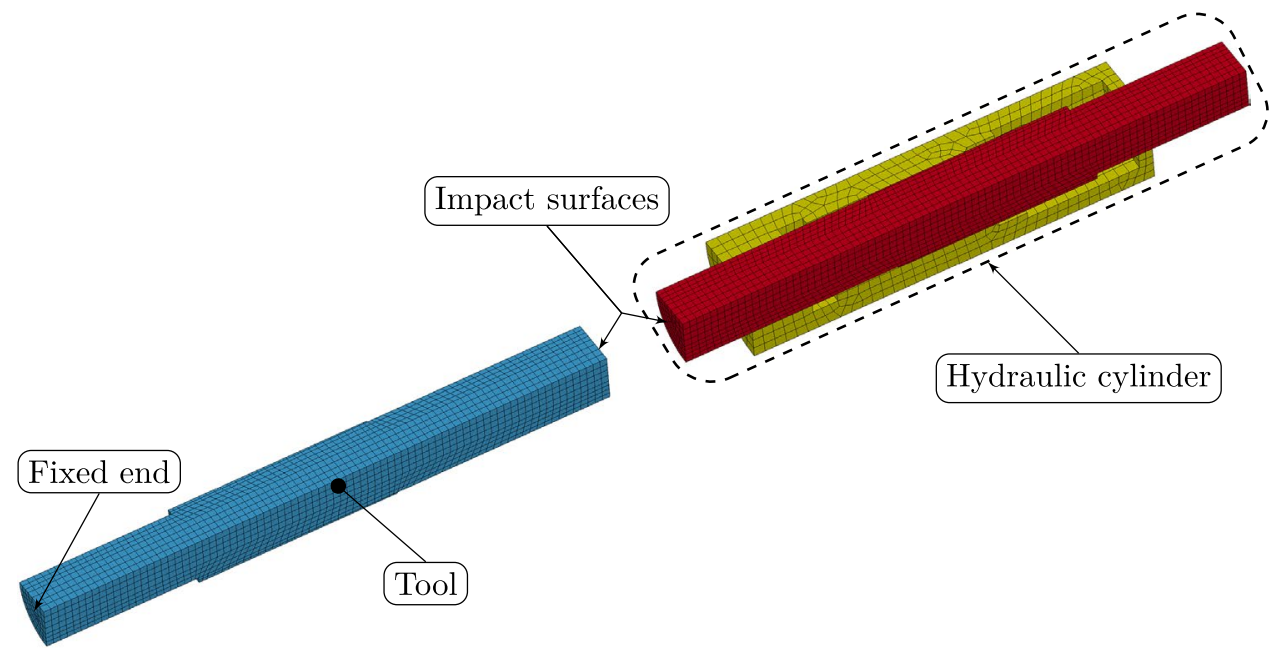

a position of $0.085 \mathrm{~m}$, the mechanical force is activated. The force will first quickly stop the piston and then accelerate it back into the cylinder housing. When the piston position is below $0.085 \mathrm{~m}$, the force is deactivated and the forces from the hydraulic pressure will control the piston movement entirely. The duration of the applied mechanical force was found to be $\sim 22 \mu$ s. The piston velocity curve, Fig. $11 \mathrm{~b}$, shows that the piston is accelerated to a velocity of $\sim 9.5 \mathrm{~m} / \mathrm{s}$ before the mechanical force is activated. Figure $11 \mathrm{c}$ shows that the pressure in Cavity A is $\sim 15 \mathrm{MPa}$ when the piston is at the inner turning point. During the acceleration phase, the pressure drops to $\sim 13 \mathrm{MPa}$ before the mechanical force is activated. At region $\mathrm{M}$, the pressure is transient and it peaks up to $19 \mathrm{MPa}$. This is due to the activation of the mechanical force and the response from the fluid system. The maximum value occurs when the piston moves into the cylinder housing and the valve is closed when changing state. In Fig. 11d, the momentary flow into Cavity A can be seen. During the acceleration phase, the flow increases to $0.008 \mathrm{~m}^{3} / \mathrm{s}$ before the mechanical force is activated, after that the flow is reversed and is decreased towards a zero flow at the inner turning point.

The magnifier in Fig. 11c shows that the curves are close to each other; a maximum time shift difference of $0.9 \mathrm{~ms}$ was noticed during the whole simulation time period. Note that the time shift varies throughout the analysis. The maximum pressure magnitude difference was estimated to $0.5 \%$, where the co-simulation pressure in general is somewhat lower compared to the reference model.

\subsection{Case 3}

The third simulation case was chosen to demonstrate the potential of the proposed method and the possibilities when evaluating deformations, stresses and strains in the structural parts. The model in Fig. 7b was used for the system simulation. The previous FE-model was updated to include a cylindrical part that represents a typical tool for a hydraulic hammer, see Fig. 12. This set-up is used to give a more realistic impact response than the mechanical force used in Case 2. The far end from the impact surface of the tool was rigidly fixed. The elastic material properties stated in Table 2 were used in the FE-model to simulate the elastic behaviour. The mesh for the tool consists of 5114 fully integrated 8-node hexahedral solid elements. The total number of elements was 12,256 . The time step used here was $10^{-7} \mathrm{~s}$, as in Case 2, resulting in a computational time of 4 hours and 44 minutes. The other parameters in the Hopsan model were also the same as in Case 2.

The results, shown in Fig. 13, indicate that the percussion unit reaches a steady-state condition after only a few working strokes. The values for the piston movement are calculated by averaging over the nodes on the piston impact surface. The piston displacement curve, Fig. 13a, shows that the piston follows the same sequence as described in Case 2. The difference is that a real impact is simulated in this case, instead of applying a mechanical force. The impact on the tool occurs at a position of $0.085 \mathrm{~m}$. A stress wave is generated at the piston impact surface at the time of impact, and the noise in the velocity curve, see Fig. 13b, after the first impact, represents the stress wave travelling back and forth in the piston. The pressure curve, Fig. 13c, is very similar to the curve in Case 2 and it follows the same pattern. The hydraulic pressure in Cavity A shows a rapid variation at the time of impact, see region $\mathrm{N}$ in Fig. 13c. At this time, the piston "bounces" on the tool and the piston moves away from the tool. This movement will cause a pressure increase in the fluid contained in Cavity A, since the piston is moving back into the cavity in a similar way as in Case 2. As the pressure increases in the cavity, the fluid force on the piston will also increase, and this will affect the piston movement. 


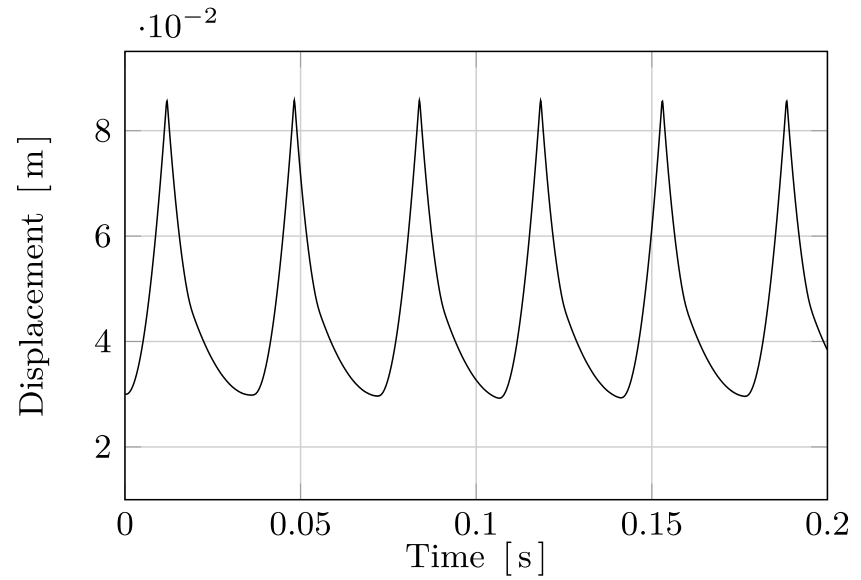

(a) Piston displacement

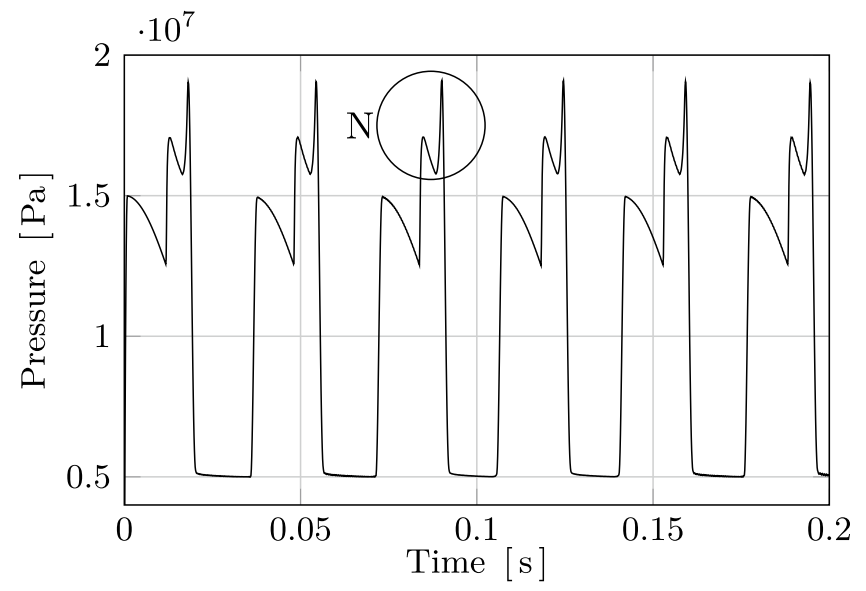

(c) Pressure in Cavity A

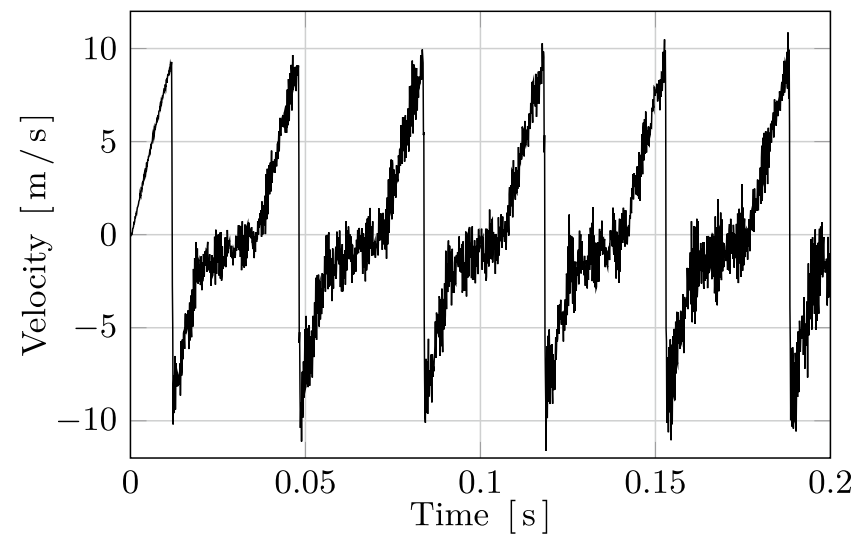

(b) Piston velocity

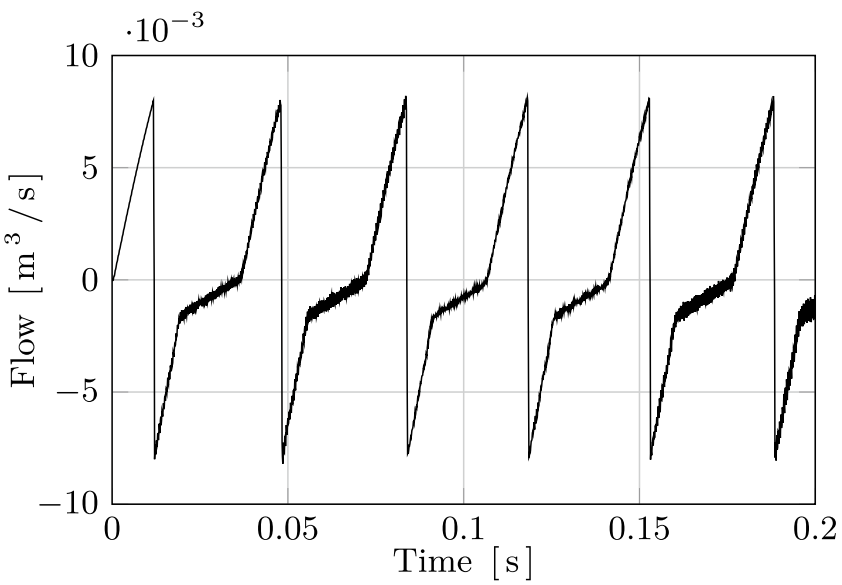

(d) Flow into Cavity A

Fig. 13 Simulation results from Case 3. Region $N$ indicates the pressure behaviour at the time of impact

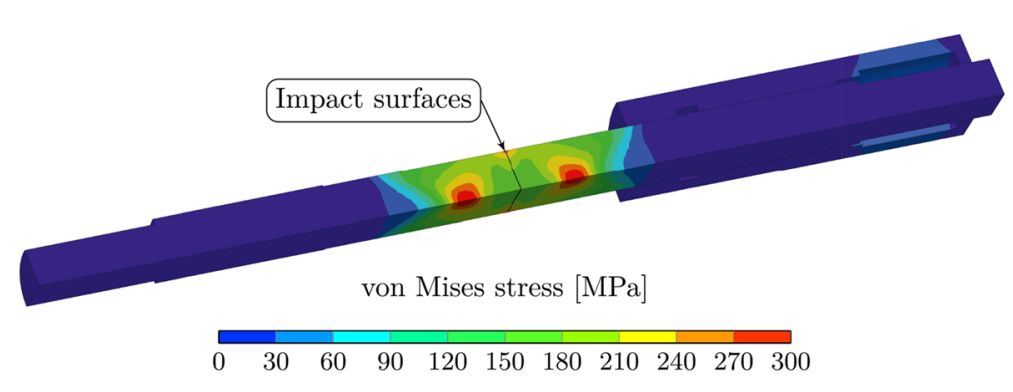

(a) Overall stress at impact

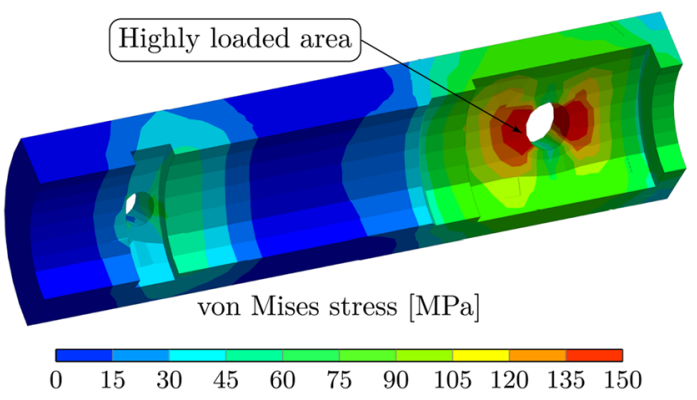

(b) Cylinder housing maximum stress

Fig. 14 FE-results from Case 3, showing a the von Mises stress in the piston and tool at the time of impact, $\mathbf{b}$ the maximum von Mises stress in the cylinder housing during the analysis, $t=159.2 \mathrm{~ms}$. Notice that different fringe-levels are used 


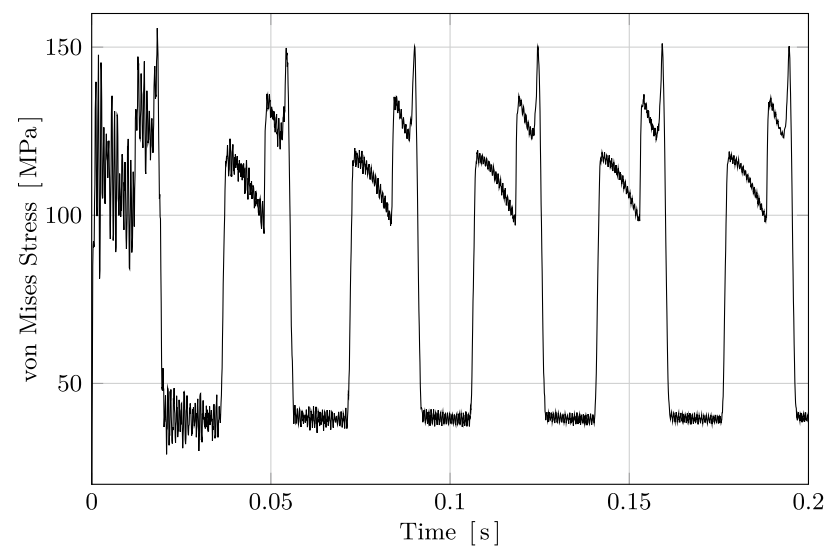

Fig. 15 Time history FE-results from Case 3. Stress results from an element at the highly loaded area, shown in Fig. 14. The load cycle in the material becomes very clear

Figures 14 and 15 show some examples of FE-results that are available from the simulation. In this case, the equivalent von Mises stress is shown, but also displacements are available. The transient stress curve in Fig. 15 exhibits the same behaviour as the pressure curve from Cavity A, cf. Fig. 13c.

\section{Discussion}

The co-simulation method using the FMI-standard that is proposed in this paper has not been found implemented for TLM and explicit non-linear FEM before. Similar published works connecting $1 \mathrm{D}$ and 3D simulation models have been found using other techniques. However, the major part of these works are not related to wave transmission over the co-simulation interface, but to heat transfer or steady-state fluid flow.

The implemented co-simulation interface generates an FMU representing the LS-DYNA FE-model. In this work, it has only been used together with Hopsan, but since the FMI standard is used, it is possible to include it in other FMI supporting tools as well. The models are coupled using the TLM method and the implementation is based on the power port variables defined by Hopsan. To make it possible to generate more general FMUs, the model-level configuration format and the FMU generator would need to be extended.

The use of TLM elements as the interface between fluid and structure introduces a parasitic inductance (extra mass) that increases quadratically with the simulation time step. However, since it is of interest to simulate wave propagation through stiff solids, the time step must be kept low anyway. Thus, the parasitic effects do not become very large in this application case.
The results from Case 1 show that the method is able to co-simulate a 1D hydraulic system model and a 3D structural FE-model in the case of a hydraulically controlled piston. The results show identical behaviour for the reference and the co-simulated model, except for a noticed small time shift that is likely caused by:

- The implementation approximation according to Eq. 4

- Numeric differences since different solving techniques are used by Hopsan and LS-DYNA. These differences will also be accumulated resulting in an increasing absolute error throughout the simulation

The parasitic inductances from the TLM-method should not affect the time shift since these are the same for both the reference and the co-simulation model. Normally five working strokes are enough to reach a steady-state condition for the hydraulic percussion unit, which gives a typical simulation time period of $0.1-0.5 \mathrm{~s}$. As observed in Sect. 4.1, the time shift is increasing over the simulation. Even if the simulated time period is increased from 0.3 to $0.5 \mathrm{~s}$, a time shift error of only $\approx 0.3 \mathrm{~ms}$ can be expected. This is normally insignificant for this application. However, the cause of the observed time shift needs to be further investigated to fully understand the limits of this method.

In Case 1, a short duration/high amplitude pressure peak arises in Cavity A, see $t \approx 0.2 \mathrm{~s}$ in Fig. 9c. At this point, the valve is closed for a short time during the change of state, and since the piston is moving into the cavity the pressure will increase. When the valve has changed state, the pressure will be relieved and adapt to the pressure source to which it is connected. This is a main mechanism for hydraulic percussion units and it is of great importance that the simulation method is able to capture this short duration effect, which this method does. The identified behaviour indicates that correct and stable results are obtained, and that short duration dynamics are resolved.

In Case 2, the method is evaluated for a short duration mechanical force applied to the FE-model, representing a more complex system than in the first case. The purpose of this procedure is to simulate an elastic contact, or rather an elastic impact, and to evaluate the co-simulation interface when there are external loads, other than the hydraulic ones, acting on the piston. A rather small time step, $T=10^{-7} \mathrm{~s}$, was used here to keep the amplitude error $\epsilon$ within an acceptable level. It was found that the duration of the mechanical force was strongly dependent of the chosen time step. If the mechanical force duration is made time step independent, the amplitude error should be at the same level as in Case 1. Compared to Case 1, the piston movement here is not that smooth, especially when the force is activated, which of course is to be expected. Furthermore, the time between the impacts shows a different behaviour 
for the velocity, pressure and flow curves. During the phase before the impact, a pure acceleration due to the pressure is obtained. The piston will stop almost immediately when the force becomes activated, which will affect pressure and flow in the fluid system. This effect can be noticed in the pressure curve as a number of short duration pressure peaks at the time of impact, region $\mathrm{M}$ in Fig. 11c. As in Case 1, this short duration transient is also captured here. A small time shift can be observed when comparison is made with the reference model. It can also be observed that the time shift varies throughout the simulated time period. As stated previously, the noticed time shift is also here insignificant for this application. Most likely the chosen modelling technique of the mechanical force will affect the observed time shift. The cause of this variation needs to be addressed to achieve an even better agreement with the reference model.

Case 3 is meant as a demonstration of the proposed method for an application closer to reality. No reference model was used for comparison here, because no deformable bodies are implemented in Hopsan. The difference from Case 2 is that the impact is simulated through contact between two elastic bodies, rather than using a mechanical force. The complexity in the fluid system is the same as before, but in the FE-simulation contact calculations must be performed, which is a more complex task than applying a mechanical force. The co-simulation coupling method will also be more stressed than in the second case, since the effects of stress waves must be handled. The results from the fluid simulation are similar to the second case, except for the flow. The noise in this curve, Fig. 13d, is caused by the stress wave that is travelling back and forth in the piston, which can also be observed in the piston velocity curve, Fig. 13b, by which the flow is calculated. The FEresults here chosen consist of deformation and stresses for the whole 3D-geometry and for the simulated time period. The third case shows that the proposed method is capable of simulating the coupled fluid-structure problem where complex dynamic properties, such as mechanical contacts and changing direction of flow, are present. The coupling between the structural and fluid dynamics that is captured in the proposed co-simulation method is difficult, or impossible, to achieve with decoupled simulation models.

\section{Concluding remarks}

This paper presents a method where 1D-fluid and 3D-structural models are co-simulated. This method is developed for handling cases when complex fluid systems are used for driving complex solid structural elements, and when the fluid structure interaction is of major importance, which is the case in hydraulic percussion units. The fluid system is modelled in a 1D-system simulation tool and the important system dynamic behaviour for the intended application is fully represented without the necessity to use 3D CFD methods, which gives a computationally very efficient fluid simulation. Conventional FE-systems can be used for the structural simulation, and full 3D results will be available to be used in stress analysis, fatigue assessment or acoustical radiation analysis. The most important contributions from this work are:

- A co-simulation method for a fluid-structure coupling has been developed

- The implemented co-simulation interface is based on the FMI-standard and TLM

- Flexible engineering-friendly automatic generation of the interface FMU module

- Computationally inexpensive 1D-system fluid simulation

- Extended simulated time period facilitated due to an efficient fluid simulation

- Short duration dynamics in both the fluid and the structural system are resolved

- Full 3D results and time history data from the structural FE-simulation are available for, e.g. stress analysis, fatigue assessment or calculation of acoustical radiation

Acknowledgments The authors would like to thank the project initiator Erik Sigfridsson and Atlas Copco Construction Tools for funding the project.

Open Access This article is distributed under the terms of the Creative Commons Attribution 4.0 International License (http://creativecommons.org/licenses/by/4.0/), which permits unrestricted use, distribution, and reproduction in any medium, provided you give appropriate credit to the original author(s) and the source, provide a link to the Creative Commons license, and indicate if changes were made.

\section{References}

1. LSTC (2015) LS-DYNA Theory manual. Livermore Software Technology Corporation, Livermore

2. ANSYS Inc (2015) ANSYS multiphysics user's guide. Canonsburg, Pennsylvania

3. Bazilevs Y, Takizawa K, Tezduyar TE (2013) Computational fluid-structure interaction: methods and applications. Wiley, Hoboken

4. Casadei F, Halleux J, Sala A, Chillè F (2001) Transient fluidstructure interaction algorithms for large industrial applications. Comput Methods Appl Mech Eng 190(24-25):3081-3110

5. Wang Y, Feng J, Zhang B, Peng X (2013) Modeling the valve dynamics in a reciprocating compressor based on two-dimensional computational fluid dynamic numerical simulation. Proc Inst Mech Eng Part E J Process Mech Eng 227(4):295-308

6. Campbell R, Paterson E (2011) Fluid-structure interaction analysis of flexible turbomachinery. J Fluids Struct 27(8):1376-1391

7. Rauch G, Lutz J, Werner M, Gurwara S, Steinberg P (2015) Synergetic 1D-3D-coupling in engine development part i: Verification of concept. Tech. rep, SAE Technical Paper 
8. Bayrasy P, Burger M, Dehning C, Kalmykov I, Speckert M (2012) Applications for MBS-FEM-coupling with MpCCI using automotive simulation as example. Proceedings of the 2nd Commercial Vehicle Technology Symposium (CVT 2012). Kaiserslautern, Germany, pp 385-394

9. Blochwitz T, Otter M, Åkesson J, Arnold M, Clauss C, Elmqvist H, Friedrich M, Junghanns A, Mauss J, Neumerkel D, Olsson H, Viel A (2012) Functional mockup interface 2.0: The standard for tool independent exchange of simulation models. In: Proceedings of the 9th International Modelica Conference, Munich, Germany, pp 173-184

10. Axin M, Braun R, Dell'Amico A, Eriksson B, Nordin P, Pettersson K, Staack I, Krus P (2010) Next generation simulation software using transmission line elements. In: Fluid Power and Motion Control, Bath, England

11. Eriksson B, Nordin P, Krus P (2010) Hopsan NG, a C++ implementation using the TLM simulation technique. In: The 51st Conference On Simulation And Modelling, Oulu, Finland

12. Krus P, Jansson A, Palmberg JO, Weddfelt K (1990) Distributed simulation of hydromechanical systems. In: The Third Bath International Fluid Power Workshop, Bath, England

13. Stavåker $\mathrm{K}$, Ronnås $\mathrm{S}$, Wlotzka $\mathrm{M}$, Heuveline $\mathrm{V}$, Fritzson P (2013) PDE modeling with Modelica via FMI import of HiFlow3 C ++ components. Proceedings of SIMS 54th Conference on Simulation and Modelling. Bergen, Norway, pp 16-23

14. Open Source Modelica Consortium (2015) The OpenModelica modeling, simulation, and software development environment. Open Source Modelica Consortium, Linköping, Sweden, URL http://www.openmodelica.org

15. Anzt $\mathrm{H}$, Augustin $\mathrm{W}$, Baumann $\mathrm{M}$, Bockelmann $\mathrm{H}$, Gengenbach T, Hahn T, Heuveline V, Ketelaer E, Lukarski D, Otzen A, Ritterbusch S, Rocker B, Ronnas S, Schick M, Subramanian C, Weiss JP, Wilhelm F (2010) Hiflow3 - a flexible and hardware-aware parallel finite element package. Preprint Series of the Engineering Mathematics and Computing Lab (06)

16. Ljubijankic M, Nytsch-Geusen C, Rädler J, Löffler M (2011) Numerical coupling of Modelica and CFD for building energy supply systems. In: Proceedings of the 8th International Modelica Conference, Citeseer, Dresden, Germany, vol 2011, pp 286-294

17. Kossel R, Tegethoff W, Bodmann M, Lemke N (2006) Simulation of complex systems using Modelica and tool coupling. In: 5th Modelica Conference, vol 2, pp 485-490
18. Wu DZ, Liu QL, Wu P, Wang LQ, Paulus T, Wang BG, Oesterle $M$ (2012) The research of $1 D / 3 D$ coupling simulation on pump and pipe system. IOP Conference Series: Earth and Environmental Science, 26th IAHR Symposium on Hydraulic Machinery and Systems 15(5)

19. Micu DA, Straface D, Farkas L, Erdelyi H, Iozsa MD, Mundo D, Donders S (2014) A co-simulation approach for crash analysis. UPB Scientific Bulletin, Series D. Mechanical Engineering 76(2)

20. Microsoft Corporation (2015) Microsoft excel. Microsoft Corporation, Redmond

21. The MathWorks Inc (2015) Matlab/Simulink User's Guide. The MathWorks Inc, Natick

22. Cresnik R, Rieser A, Schluder H (2009) Dynamic simulation of mechatronic systems. In: 7th European LS-Dyna Conference, Graz, Austria

23. Auslander DM (1968) Distributed system simulation with bilateral delay-line models. J Basic Eng 90(2):195-200

24. Siemers A, Fritzson D, Nakhimovski I (2009) General metamodel based co-simulations applied to mechanical systems. Simul Model Pract Theory 17(4):612-624

25. Larsson J, Krus P, Palmberg JO (2001) Modelling, simulation and validation of complex fluid and mechanical systems. In: 5th International Conference on Fluid Power Transmission and Control, pp 338-341

26. Braun R, Ericsson L, Krus P (2015) Full vehicle simulation of forwarder with semi active suspension using co-simulation. In: ASME/BATH 2015 Symposium on Fluid Power and Motion Control, Chicago, USA

27. Larsson J, Krus P (2003) Stability analysis of coupled simulation. In: ASME 2003 International Mechanical Engineering Congress and Exposition, vol 1, pp 861-868

28. Krus P, Weddfelt K, Palmberg JO (1994) Fast pipeline models for simulation of hydraulic systems. J Dyn Syst Meas Control $116: 132-136$

29. Viersma TJ (1980) Analysis, synthesis and design of hydraulic servosystems and pipelines. Elsevier Scientific Publishing Company, Amsterdam

30. Belytschko T, Liu WK, Moran B, Elkhodary K (2013) Nonlinear finite elements for continua and structures. Wiley, Hoboken 